\title{
Effects of Single-Task Versus Dual-Task Training on Balance Performance in Elderly Patients With Knee Osteoarthritis
}

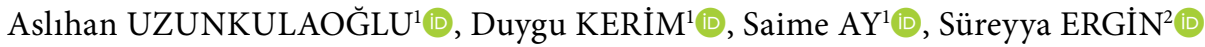 \\ ${ }^{1}$ Department of Physical Medicine and Rehabilitation, Ufuk University Faculty of Medicine, Ankara, Turkey \\ ${ }^{2}$ Department of Physical Medicine and Rehabilitation, Fizyocare Physical Medicine and Rehabilitation Center, Ankara, Turkey
}

\begin{abstract}
Objectives: This study aims to compare the effects of single-task and dual-task training on balance performance in elderly patients with knee osteoarthritis (OA).

Patients and methods: Fifty elderly osteoarthritic patients with balance impairment (16 males, 34 females; mean age $72.9 \pm 5.5$ years; range 65 to 84 years) were included in this study. Patients were randomly assigned to single-task balance training (group 1) or dual-task balance training (group 2) groups. Balance activities were given to both groups for three times a week for four weeks. Patients in group 2 also performed cognitive tasks simultaneously with these exercises. Patients were evaluated with Berg balance scale (BBS), kinesthetic ability trainer static and dynamic scores, timed up and go (TUG) test and walking speed (WS) for single and dual tasks, number of stopping and activities-specific balance confidence $(A B C)$ scale at baseline and at the end of four weeks.

Results: At the end of the therapy, there were statistically significant improvements in BBS, KAT 2000 static and dynamic scores, TUG test and WS for single and dual tasks, number of stopping and $A B C$ scale in both groups $(p<0.05)$. But there was no statistical difference in any parameter between the groups $(p>0.05)$.

Conclusion: Both single- and dual-task trainings are effective in improving balance performance under single- and dual-task conditions in elderly patients with knee OA. Dual-task training is not superior to single-task training for balance improvement in elderly osteoarthritic patients.

Keywords: Balance training, elderly, osteoarthritis.
\end{abstract}

Osteoarthritis (OA) of the knee is the most common cause of knee pain and osteoarthritic changes not only in the tissues within the articular cavity, but also the ligaments, tendons, muscles and periarticular tissues. ${ }^{1}$ There are some neuromuscular adaptations relevant with knee $\mathrm{OA}$; reduced strength and proprioceptive accuracy of the quadriceps muscle can be seen. ${ }^{2,3}$ People with knee OA have also been reported to have reduced balance, evidenced by a higher incidence of falls and increased postural sway., ${ }^{4,5}$ Altered muscle activation patterns including increased activity and co-contraction of thigh muscles during the stance phase of gait cause balance deficits. ${ }^{6,7}$ On the other hand, cognitive processing plays an important role in balance and gait and this relationship may be explained by the fact that higher order cognitive functions such as executive functions are called upon while walking. ${ }^{8}$ The decline of balance control can lead to fall injuries that result in serious physical, psychological, and social loss. An effective evaluation of balance control in knee OA patients is necessary before management of this condition. Therefore, in this study, we aimed to compare the effects of single-task and dual-

Received: September 12, 2018 Accepted: March 11, 2019 Published online: April 26, 2019

Correspondence: Aslıhan Uzunkulaoğlu, MD. Ufuk Üniversitesi Tıp Fakültesi Fiziksel Tıp ve Rehabilitasyon Anabilim Dalı, 06836 Gölbaşı, Ankara, Turkey. Tel: +90 533 - 5197927 e-mail: aslihanseyrek@gmail.com 
task training on balance performance in elderly patients with knee OA.

\section{PATIENTS AND METHODS}

This randomized controlled trial with a blind assessor was conducted in Ufuk University Faculty of Medicine between November 2015 and March 2016. The study protocol was approved by the Ufuk University Faculty of Medicine Ethics Committee. The registration number for the study is 30112015-5. A written informed consent was obtained from each patient. The study was conducted in accordance with the principles of the Declaration of Helsinki.

Fifty elderly osteoarthritic patients with balance impairment (16 males, 34 females; mean age $72.9 \pm 5.5$ years; range 65 to 84 years) were recruited in the study. Determination of OA was based on American College of Rheumatology criteria. ${ }^{5}$ Demographic and clinical characteristics of patients including medications were recorded. Inclusion criteria included age $\geq 65$, ability to walk $10 \mathrm{~m}$, having no neurological or musculoskeletal diagnosis, meeting the criteria of balance impairment, and scoring $>19$ on the mini mental state examination. Physical examination of all patients and weight-bearing radiographs were performed. Subjects whose full blood count, erythrocyte sedimentation rate (ESR), and C-reactive protein (CRP) and those with normal liver and renal function tests were recruited. Individuals were excluded if they had neurologic or musculoskeletal diagnosis such as stroke, orthopedic involvement, significant visual or auditory impairments, severe vitamin B12 deficiency or sedative drug use.

By using the website randomizer.org method, participants were randomly assigned into either the single-task balance training (group 1, $\mathrm{n}=25$ ) group or the dual-task balance training (group 2, $\mathrm{n}=25$ ) group. ${ }^{9}$ All patients were blinded to treatment allocation while the physiotherapist who applied the therapy was aware of the procedure.

Individualized training sessions of 45 minutes were given to all participants in both groups three times a week for four weeks. Group 1 received only balance exercises under single-task conditions. These balance exercises included tandem stand, semi-tandem stand, one- and twolegged stand for support, dynamic movements as tandem walk and circle turns to disturb the center of gravity, heel or toe stands for postural muscle groups and exercises to reduce sensory input like standing with eyes closed by recommendations from the American College of Sports Medicine. ${ }^{10}$

Group 2 received the same balance exercises as group 1 also under single-task conditions and they also performed cognitive tasks simultaneously with these exercises three times a week for four weeks. Cognitive tasks included singing a song, counting backwards from 10 , and counting the days of the week. ${ }^{11}$

Patients were evaluated with Berg balance scale (BBS), kinesthetic ability trainer (KAT 2000; OEM Medical, Carlsbad, USA) static and dynamic scores, timed up and go (TUG) test and walking speed (WS) for single and dual tasks, number of stopping and activities-specific balance confidence $(\mathrm{ABC})$ scale at baseline and at the end of four weeks. The BBS consists of 14 items related with daily living activities. ${ }^{12}$ Patients were asked to perform tasks about static, dynamic and functional balance. Scores in BBS were scaled from 0 , failure to perform task, to 4 , ability to perform task independently and safely, the sum of the scores being maximum $56 .{ }^{12}$ The validity and reliability study of the Turkish version of BBS was performed by Sahin et al. ${ }^{13}$

The KAT 2000 device (OEM Medical, Carlsbad, USA) consists of two main components: a moving platform and a tilt sensor. These components are connected to a computer which registers the deviation of the platform from a reference position 18.2 times each second. Patients were asked to cross their arms on their chest to prevent the contribution of arms on balance. Without changing the position of feet, the patient can tilt the platform to all directions for maintaining balance. Also, a symbol as a red " $\mathrm{X}$ " mark gives feedback about the balance on the screen of the computer. Patients were asked to stabilize the red $\mathrm{X}$ mark at the center of the screen during the static balance tests. When the dynamic test was performed, the patient was asked to superimpose the red $\mathrm{X}$ mark onto the moving cursor which drew a circle on the screen. Before recording the scores, patients were allowed to get accustomed to the device by practicing. Final score was 
calculated with the best of three scores. Higher scores indicate a poor balance performance. ${ }^{14}$

The TUG test assesses mobility. In this test, patients were seated on a back-supported chair. Then, they were asked to stand up, walk ( $3 \mathrm{~m}$ or 10 feet away), turn, walk back to the chair and sit down. Time taken to complete the test was measured in seconds. ${ }^{15}$

The WS is a valid, reliable, sensitive measure appropriate for assessing and monitoring functional status and overall health in a wide range of populations. ${ }^{16}$ There are a variety of testing protocols for assessing WS. Because a distance of $10 \mathrm{~m}$ is a more reliable option, we chose this protocol and patients walked $10 \mathrm{~m}$ at a speed at which they felt comfortable. At $6 \mathrm{~m}$, we recorded the time with a chronometer as WS. WS was recorded for all patients during both single- and dual-task conditions. Also, the number of stopping was recorded for all patients.

The ABC scale short form, developed by Powell and Myers, assesses fear of falling; contains tasks related to indoor and outdoor daily living activities, to measure balance confidence in elderly people who have various levels of functioning. ${ }^{17,18}$ Scores range from $0 \%$ (no confidence) to 100\% (complete confidence) for each question item. Higher scores indicate greater confidence. ${ }^{17}$ The validity and reliability study of the Turkish version of this scale was conducted by Kibar et al. ${ }^{19}$
The sample size and power calculations were performed using a G*Power of 3.1.9.2c power analysis program (Heinrich-Heine-Universität Düsseldorf, Germany). Due to the previous studies' definition of a clinically significant difference reduction in BBS as four units and a standard deviation (SD) of 2.90 in elderly population, and the probability of a type I error of 0.05 and a power of 0.90 , the estimated sample size for each group was 23. We recruited 25 patients for each group because of the possibility that a small number of patients would drop out over the course of the study.

\section{Statistical analysis}

All analyses were performed using the PASW for Windows version 18.0 software (SPSS Inc., Chicago, IL, USA). The means and SDs were given as descriptive statistics. All data for normality were tested using KolmogorovSmirnov test. Wilcoxon test was used to calculate the pre- and post-treatment value differences. Mann-Whitney $\mathrm{U}$ was used to compare the differences between two groups. A level of significance of $p<0.05$ was accepted.

\section{RESULTS}

All patients completed the study protocol and none had any side effects. The results of full blood count, ESR, CRP and biochemical markers

Table 1. Demographic characteristics and baseline values of outcome measures

\begin{tabular}{|c|c|c|c|c|c|}
\hline & \multicolumn{2}{|c|}{ Group $1(\mathrm{n}=25)$} & \multicolumn{2}{|c|}{ Group $2(n=25)$} & \multirow[b]{2}{*}{$p$} \\
\hline & $\mathrm{n}$ & Mean \pm SD & $\mathrm{n}$ & Mean \pm SD & \\
\hline Age (year) & & $73.6 \pm 5.6$ & & $72.3 \pm 5.5$ & 0.442 \\
\hline $\begin{array}{l}\text { Sex } \\
\quad \text { Female } \\
\text { Male }\end{array}$ & $\begin{array}{c}16 \\
9\end{array}$ & & $\begin{array}{c}18 \\
7\end{array}$ & & 0.548 \\
\hline Berg balance scale & & $30.5 \pm 6.1$ & & $29.4 \pm 5.4$ & 0.521 \\
\hline Kinesthetic ability trainer 2000 static score & & $1468.1 \pm 466.6$ & & $1528.4 \pm 474.1$ & 0.712 \\
\hline Kinesthetic ability trainer 2000 dynamic score & & $2006.6 \pm 447.4$ & & $2020.6 \pm 441.7$ & 0.869 \\
\hline Timed up and go single-task & & $13.2 \pm 4.3$ & & $13.7 \pm 4.4$ & 0.463 \\
\hline Timed up and go dual-task & & $15.3 \pm 5.1$ & & $15.8 \pm 5.1$ & 0.564 \\
\hline Walking speed single-task & & $15.1 \pm 4.7$ & & $16.2 \pm 4.9$ & 0.311 \\
\hline Walking speed dual-task & & $17.0 \pm 5.5$ & & $18.2 \pm 5.6$ & 0.442 \\
\hline Number of stopping & & $1.0 \pm 0.8$ & & $1.4 \pm 1.2$ & 0.167 \\
\hline Activities-specific balance confidence scale & & $50.5 \pm 17.0$ & & $50.8 \pm 18.9$ & 0.729 \\
\hline
\end{tabular}


Table 2. Comparison of outcome measures in and between groups

\begin{tabular}{|c|c|c|c|c|c|c|c|}
\hline & \multicolumn{3}{|c|}{ Group 1} & \multicolumn{3}{|c|}{ Group 2} & \multirow{3}{*}{$\frac{\text { Group 1-2 }}{p}$} \\
\hline & BT & AT & & BT & AT & & \\
\hline & Mean \pm SD & Mean \pm SD & $p$ & Mean \pm SD & Mean \pm SD & $p$ & \\
\hline BBS & $30.5 \pm 6.1$ & $34.5 \pm 6.3$ & $<0.001^{*}$ & $29.4 \pm 5.4$ & $34.8 \pm 5.7$ & $<0.001^{*}$ & 0.69 \\
\hline KAT 2000 static score & $1468.1 \pm 466.6$ & $1167.3 \pm 468.0$ & $<0.001^{*}$ & $1528.4 \pm 474.1$ & $1232.9 \pm 487.5$ & $<0.001^{*}$ & 0.62 \\
\hline KAT 2000 dynamic score & $2006.6 \pm 447.4$ & $1683.8 \pm 450.0$ & $<0.001^{*}$ & $2020.6 \pm 441.7$ & $1567.8 \pm 455.0$ & $<0.001^{*}$ & 0.47 \\
\hline TUG single-task & $13.2 \pm 4.3$ & $11.2 \pm 4.3$ & $<0.001^{*}$ & $13.7 \pm 4.4$ & $11.6 \pm 4.3$ & $<0.001^{*}$ & 0.53 \\
\hline TUG dual-task & $15.3 \pm 5.1$ & $14.3 \pm 5.1$ & $<0.001^{*}$ & $15.8 \pm 5.1$ & $12.6 \pm 5.0$ & $<0.001^{*}$ & 0.09 \\
\hline Walking speed single-task & $15.1 \pm 4.7$ & $13.2 \pm 4.8$ & $<0.001^{*}$ & $16.2 \pm 4.9$ & $13.7 \pm 4.8$ & $<0.001^{*}$ & 0.80 \\
\hline Walking speed dual-task & $17.0 \pm 5.5$ & $15.9 \pm 5.5$ & $<0.001^{*}$ & $18.2 \pm 5.6$ & $15.0 \pm 5.4$ & $<0.001^{*}$ & 0.41 \\
\hline Number of stopping & $1.0 \pm 0.8$ & $0.5 \pm 0.7$ & $0.01^{*}$ & $1.4 \pm 1.2$ & $0.6 \pm 0.6$ & $<0.001^{*}$ & 0.36 \\
\hline ABC scale & $50.5 \pm 17.0$ & $60.5 \pm 17.0$ & $<0.001^{*}$ & $50.8 \pm 18.9$ & $55.8 \pm 18.9$ & $<0.001^{*}$ & 0.07 \\
\hline
\end{tabular}

BT: Before treatment; AT: After treatment; SD: Standard deviation; BBS: Berg balance scale; KAT 2000: Kinesthetic ability trainer; TUG: Timed up and go; ABC: Activities-specific balance confidence; * $\mathrm{p}<0.05$.

were in normal ranges for both groups. Of the patients, 31 had Kellgren-Lawrence grade $3 \mathrm{OA}$ and 19 had Kellgren-Lawrence grade 4 OA.

Patients' demographic characteristics and baseline values of the outcome measures are presented in Table 1. There were no statistically significant differences between the groups for baseline values $(p>0.05)$ (Table 1$)$.

Statistically significant improvements were observed in BBS $(p<0.001$ for both groups), KAT 2000 static $(p<0.001$ for both groups) and dynamic scores ( $p<0.001$ for both groups), TUG for single ( $p<0.001$ for both groups) and dual tasks ( $<<0.001$ for both groups), WS for single ( $p<0.001$ for both groups) and dual tasks $(p<0.001$ for both groups), number of stopping ( $p=0.01$ for group 1 and $p<0.001$ for group 2) and $A B C$ scale $(p<0.001$ for both groups) for both groups at the end of the therapy $(p<0.05)$ (Table 2).

At the end of the therapy, there was no statistical difference in BBS $(p=0.69)$, KAT 2000 static $(p=0.62)$ or dynamic scores $(p=0.47)$, TUG for single $(p=0.53)$ or dual tasks $(p=0.09)$, WS for single $(p=0.80)$ or dual tasks $(p=0.41)$, number of stopping $(p=0.36)$ or ABC scale $(p=0.07)$ between the groups (Table 2).

\section{DISCUSSION}

The aim of this study was to compare the effects of single-task and dual-task training on balance performance in elderly osteoarthritic patients with balance impairment. There are some studies about the effects of dual-task training on balance in varying conditions. Konak et al. ${ }^{20}$ conducted a study with 42 older adults with balance impairment and osteoporosis and found that four-week single- or dual-task balance exercise programs are effective in improving balance. They also revealed that the application of a specific type of dual-task exercise program provides greater improvement. Our results were consistent with this study while we detected no significant difference for WS or $\mathrm{ABC}$ scale results after the application of the dual-task exercise program. This result can be due to our study population. We conducted this study with osteoarthritic patients and mechanisms for balance impairment for $\mathrm{OA}$ are different from osteoporosis. Also, as a power of our work, our study population was larger. On the other hand, similar to our results, a study by Targino et al. ${ }^{21}$ with six pre-frail elderly patients demonstrated that dual-task performance had no additional value in relation to the improvement of balance in general.

Walking speed is an important measure of functional capacity among the elderly. ${ }^{22}$ In a study by Silsupadol et al., ${ }^{23}$ older adults with balance impairment were recruited and randomized into three groups (single- or dualtask training groups with fixed or variable priority instructions) to be evaluated for WS and 
balance. Authors found that dual-task training with variable priority instructions can be effective than single-task training in improving WS in elderly participants with balance impairment. There were no significant differences between the groups for balance evaluated by BBS. In our study, we found no significant difference in terms of WS between the groups. We evaluated balance with BBS, ABC scale, and KAT 2000 device as Silsupadol et al. ${ }^{23}$ however, we found no superiority for dual-task training compared to single-task training. These results can be explained by our population's OA and pain, which affect WS negatively. On the other hand, we evaluated WS at both single- and dualtask conditions for both groups. Furthermore, Silsupadol et al.'s study ${ }^{23}$ population was so small that their results can be interpreted as controversial.

This study has some limitations. First of all, we could only conduct a four-week exercise program. However, it is well known that balance exercises for a prolonged intervention have more positive effects for physical activity. ${ }^{24}$ Secondly, under the dual-task condition, prioritizing one task determines the direction of attention to tasks; so, this can affect the dual-task performance. Also, difficulty of tasks can vary between subjects and affect the results. Thirdly, we did not evaluate patients in terms of obesity while it is well known that body mass index is a major performance determinant of both static and dynamic balance tests. $^{25}$

In conclusion, both single- and dual-task trainings are effective in improving balance performance under single- or dual-task conditions in elderly osteoarthritic patients. Dual-task training is not superior to single-task training for balance improvement in elderly osteoarthritic patients. Long-term studies are needed with tasks chosen according to the capacity of the subjects objectively.

\section{Declaration of conflicting interests}

The authors declared no conflicts of interest with respect to the authorship and/or publication of this article.

\section{Funding}

The authors received no financial support for the research and/or authorship of this article.

\section{REFERENCES}

1. Hermens HJ, Freriks B, Disselhorst-Klug C, Rau G. Development of recommendations for SEMG sensors and sensor placement procedures. J Electromyogr Kinesiol 2000;10:361-74.

2. Kim BR, Choi KH, Chun MH, Lee MC, Chung SJ, Jang KW. Evaluation of balance control in patients with idiopathic Parkinson's disease using tetra-ataxiometric posturography. J Korean Acad Rehab Med 2009;33:538-46.

3. Masui T, Hasegawa Y, Yamaguchi J, Kanoh T, Ishiguro N, Suzuki S. Increasing postural sway in rural-community-dwelling elderly persons with knee osteoarthritis. J Orthop Sci 2006;11:353-8.

4. Thorp LE, Sumner DR, Block JA, Moisio KC, Shott S, Wimmer MA. Knee joint loading differs in individuals with mild compared with moderate medial knee osteoarthritis. Arthritis Rheum 2006;54:3842-9.

5. Altman R, Asch E, Bloch D, Bole G, Borenstein $\mathrm{D}$, Brandt $\mathrm{K}$, et al. Development of criteria for the classification and reporting of osteoarthritis. Classification of osteoarthritis of the knee. Diagnostic and Therapeutic Criteria Committee of the American Rheumatism Association. Arthritis Rheum 1986;29:1039-49.

6. Duffell LD, Southgate DF, Gulati V, McGregor AH. Balance and gait adaptations in patients with early knee osteoarthritis. Gait Posture 2014;39:1057-6.

7. Berg K, Wood-Dauphinee S, Williams JI. The Balance Scale: reliability assessment with elderly residents and patients with an acute stroke. Scand J Rehabil Med 1995;27:27-36.

8. Smith-Ray RL, Hughes SL, Prohaska TR, Little DM, Jurivich DA, Hedeker D. Impact of Cognitive Training on Balance and Gait in Older Adults. J Gerontol B Psychol Sci Soc Sci 2015;70:357-66.

9. Available from: www.randomizer.org

10. Chodzko-Zajko WJ, Proctor DN, Fiatarone Singh MA, Minson CT, Nigg CR, Salem GJ, et al. American College of Sports Medicine position stand. Exercise and physical activity for older adults. Med Sci Sports Exerc 2009;41:1510-30.

11. Silsupadol P, Siu KC, Shumway-Cook A, Woollacott $\mathrm{MH}$. Training of balance under single- and dual-task conditions in older adults with balance impairment. Phys Ther 2006;86:269-81.

12. Berg KO, Maki BE, Williams JI, Holliday PJ, WoodDauphinee SL. Clinical and laboratory measures of postural balance in an elderly population. Arch Phys Med Rehabil 1992;73:1073-80.

13. Sahin F, Yilmaz F, Ozmaden A, Kotevolu N, Sahin T, Kuran B. Reliability and validity of the Turkish version of the Berg Balance Scale. J Geriatr Phys Ther 2008;31:32-7.

14. Hansen MS, Dieckmann B, Jensen K, Jakobsen $\mathrm{BW}$. The reliability of balance tests performed on the 
kinesthetic ability trainer (KAT 2000). Knee Surg Sports Traumatol Arthrosc 2000;8:180-5.

15. Shumway-Cook A, Brauer S, Woollacott M. Predicting the probability for falls in community-dwelling older adults using the Timed Up \& Go Test. Phys Ther 2000;80:896-903.

16. Middleton A, Fritz SL, Lusardi M. Walking speed: the functional vital sign. J Aging Phys Act 2015;23:314-22.

17. Powell LE, Myers AM. The Activities-specific balance confidence (ABC) scale. J Gerontol A Biol Sci Med Sci 1995;50:28-34.

18. Schepens S, Goldberg A, Wallace M. The short version of the Activities-specific Balance Confidence $(\mathrm{ABC})$ scale: its validity, reliability, and relationship to balance impairment and falls in older adults. Arch Gerontol Geriatr 2010;51:9-12.

19. Kibar S, Konak HE, Ay S, Evcik D, Ergin S. Turkish translation and validation of the short version of the Activities-specific Balance Confidence (ABC) scale in geriatric population. 25th National Physical Medicine and Rehabilitation Congress; Apr 22-26, 2015; Antalya, Turkey; 2015. p. 342.

20. Konak HE, Kibar S, Ergin ES. The effect of singletask and dual-task balance exercise programs on balance performance in adults with osteoporosis: a randomized controlled preliminary trial. Osteoporos Int 2016;27:3271-8.

21. Targino VR, Freire ANF, Sousa ACPA, Maciel NFB, Guerra RO. Effects of a dual-task training on dynamic and static balance control of pre-frail elderly: a pilot study. Fisioter Mov 2012;25:351-60.

22. Busch Tde A, Duarte YA, Pires Nunes D, Lebrão ML, Satya Naslavsky M, dos Santos Rodrigues A, et al. Factors associated with lower gait speed among the elderly living in a developing country: a cross-sectional population-based study. BMC Geriatr 2015;15:35.

23. Silsupadol P, Shumway-Cook A, Lugade V, van Donkelaar P, Chou LS, Mayr U, et al. Effects of singletask versus dual-task training on balance performance in older adults: a double-blind, randomized controlled trial. Arch Phys Med Rehabil 2009;90:381-7.

24. de Labra C, Guimaraes-Pinheiro C, Maseda A, Lorenzo T, Millán-Calenti JC. Effects of physical exercise interventions in frail older adults: a systematic review of randomized controlled trials. BMC Geriatr 2015;15:154.

25. Manckoundia P, Buatois S, Gueguen R, PerretGuillaume C, Laurain MC, Pfitzenmeyer P, et al. Clinical determinants of failure in balance tests in elderly subjects. Arch Gerontol Geriatr 2008;47:217-28. 